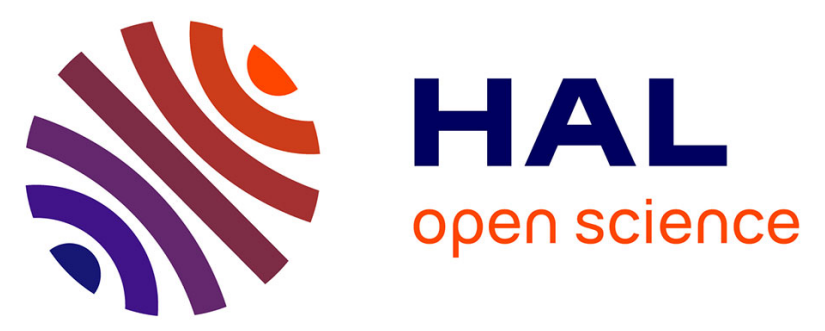

\title{
Monolithic Integration of Diluted-Nitride III-V-N Compounds on Silicon Substrates: Toward the III-V/Si Concentrated Photovoltaics
}

Olivier Durand, Samy Almosni, Yanping Wang, C. Cornet, A. Létoublon, Cédric Robert Robert, Christophe Levallois, L. Pedesseau, Alain Rolland, J. Even, et al.

\section{To cite this version:}

Olivier Durand, Samy Almosni, Yanping Wang, C. Cornet, A. Létoublon, et al.. Monolithic Integration of Diluted-Nitride III-V-N Compounds on Silicon Substrates: Toward the III-V/Si Concentrated Photovoltaics. Energy Harvesting and Systems, 2014, 1 (3-4), pp.147-156. 10.1515/ehs-2014-0008 . hal-01166477

\section{HAL Id: hal-01166477 https://hal.science/hal-01166477}

Submitted on 22 Jun 2015

HAL is a multi-disciplinary open access archive for the deposit and dissemination of scientific research documents, whether they are published or not. The documents may come from teaching and research institutions in France or abroad, or from public or private research centers.
L'archive ouverte pluridisciplinaire HAL, est destinée au dépôt et à la diffusion de documents scientifiques de niveau recherche, publiés ou non, émanant des établissements d'enseignement et de recherche français ou étrangers, des laboratoires publics ou privés. 


\section{Special Issue Article}

O. Durand*, S. Almosni, Y. Ping Wang, C. Cornet, A. Létoublon, C. Robert, C. Levallois, L. Pedesseau, A. Rolland, J. Even, J.M. Jancu, N. Bertru, A. Le Corre, F. Mandorlo, M. Lemiti, P. Rale, L. Lombez, J.-F. Guillemoles, S. Laribi, A. Ponchet and J. Stodolna

\section{Monolithic Integration of Diluted-Nitride III-V-N Compounds on Silicon Substrates: Toward the III-V/Si Concentrated Photovoltaics}

\begin{abstract}
GaAsPN semiconductors are promising material for the development of high-efficiency tandem solar cells on silicon substrates. GaAsPN diluted-nitride alloy is studied as the top-junction material due to its perfect lattice matching with the Si substrate and its ideal bandgap energy allowing a perfect current matching with the $\mathrm{Si}$ bottom cell. The $\mathrm{GaP} / \mathrm{Si}$ interface is also studied in order to obtain defect-free GaP/Si pseudo-substrates suitable for the subsequent GaAsPN top junctions growth. Result shows that a double-step growth procedure suppresses
\end{abstract}

\footnotetext{
*Corresponding author: 0. Durand, UMR FOTON, CNRS, INSA de Rennes, F-35708 Rennes, France, E-mail:

olivier.durand@insa-rennes.fr
}

S. Almosni: E-mail: samy.almosni1@insa-rennes.fr, Y. Ping Wang: E-mail: Yanping.Wang@insa-rennes.fr, C. Cornet: E-mail: Charles.Cornet@insa-rennes.fr, A. Létoublon: E-mail: Antoine.Letoublon@insa-rennes.fr, UMR FOTON, CNRS, INSA de Rennes, F-35708 Rennes, France

C. Robert, Tyndall National Institute, Cork, Ireland, E-mail: cedric.robert@tyndall.ie

C. Levallois: E-mail: Christophe.levallois@insa-rennes.fr,

L. Pedesseau: E-mail: laurent.pedesseau@insa-rennes.fr,

A. Rolland: E-mail: alain.rolland@univ-rennes1.fr, J. Even:

E-mail: jacky.even@insa-rennes.fr, J.M. Jancu:

E-mail: Jean-Marc.Jancu@insa-rennes.fr, N. Bertru:

E-mail: nicolas.bertru@insa-rennes.fr, A. Le Corre:

E-mail: alain.le-corre@insa-rennes.fr, UMR FOTON, CNRS, INSA de Rennes, F-35708 Rennes, France

F. Mandorlo: E-mail: fabien.mandorlo@insa-lyon.fr, M. Lemiti: E-mail: mustapha.lemiti@insa-lyon.fr, INL, INSA de Lyon, Bâtiment Blaise Pascal, 7 Avenue Jean Capelle, 69621 Villeurbanne Cedex, France

P. Rale: E-mail: pierre-externe.rale@edf.fr, L. Lombez: E-mail: laurent.lombez@chimie-paristech.fr, J.-F. Guillemoles: E-mail: jf-guillemoles@chimie-paristech.fr, S. Laribi: E-mail: sana.laribi@edf.fr, Institut de Recherche et Développement sur l'Energie Photovoltaïque (IRDEP), UMR 7174 - CNRS-EDF-ENSCP, EDF R\&D, 6 quai Watier, 78401 Chatou Cedex, France

A. Ponchet: E-mail: anne.ponchet@cemes.fr, J. Stodolna: E-mail: julien.stodolna@cemes.fr, CEMES-CNRS, Université de Toulouse, 29 rue J. Marvig BP 94347, 31055 Toulouse Cedex 4, France most of the microtwins and a bi-stepped Si buffer can be grown, suitable to reduce the anti-phase domains density. We also review our recent progress in materials development of the GaAsPN alloy and our recent studies of all the different building blocks toward the development of a PIN solar cell. GaAsPN alloy with energy bandgap around $1.8 \mathrm{eV}$, lattice matched with the Si substrate, has been achieved. This alloy displays efficient photoluminescence at room temperature and good light absorption. An earlystage GaAsPN PIN solar cell prototype has been grown on a $\mathrm{GaP}(001)$ substrate. The external quantum efficiency and the $I-V$ curve show that carriers have been extracted from the GaAsPN alloy absorber, with an open-circuit voltage above $1 \mathrm{eV}$, however a low short-circuit current density obtained suggests that GaAsPN structural properties need further optimization. Considering all the pathways for improvement, the 2.25\% efficiency and IQE around 35\% obtained under AM1.5G is however promising, therefore validating our approach for obtaining a lattice-matched dual-junction solar cell on silicon substrate.

Keywords: III-V semiconductors, dilute nitride, molecular beam epitaxy, multijunction solar cells, silicon

DOI 10.1515/ehs-2014-0008

\section{Introduction}

To date, the highest efficiency conversions have been reached by using III-V monocrystalline multijunction solar cell (MJSC) under concentrated sunlight. Spire Semiconductors have pushed solar cell record to $42.3 \%$, in AM1.5D conditions, for terrestrial applications, with a metamorphic (lattice-mismatched) GaInP/GaAs/GaInAs triple junction under solar concentration of 406 suns, and using a lightly doped n-type GaAs wafer. Moreover, 
a III-V triple junction coherently grown (lattice matched) onto GaAs substrate has been performed by Solar Junction. This solar cell has shown a $44 \%$ efficiency under 947 suns (AM1.5D spectra) (Derkacs et al. 2012) and contains a highly rewarded $1 \mathrm{eV}$ GaInAsNSb diluted-nitride junction. However, maintaining the GaAs, or Ge, substrates to build these high-efficiency III-V solar cells, undoubtedly incurs a substantial cost associated with such substrates. To realize the strategic challenge of cost of $0.25-0.5$ Euro/Wp, we have chosen to use the abundantly available on earth, and therefore lowcost, silicon material as a substrate. Indeed, a true monolithic integration of the III-V compound semiconductor heterostructures with silicon is receiving great interest since it will enable simultaneous both high-efficiency and low-cost production. Moreover, this approach benefits from the high market throughput capabilities of the silicon industry that is superior to any other material.

A tandem solar cell, made of a $1.7 \mathrm{eV}$ III-V top and a $1.1 \mathrm{eV}$ crystalline silicon (c-Si) bottom cell, would theoretically reach an efficiency of $37 \%$, under an AM $1.5 \mathrm{G}$ (Kurtz, Faine, and Olson 1990). However, efficiency of MJSC is very sensitive to the structural defects such as misfit dislocations, appearing during metamorphic growth, since they dramatically reduce the carrier lifetime, and thus the current extraction, and therefore reduction of the solar cell performance. Therefore, combination of both the III-V and Si technologies through a perfect lattice-matched epitaxial PV structure on silicon substrate would allow increasing significantly the efficiency, as well as reducing the overall cost of the PV multijunction cell.

Considering a perfect lattice matching, the first challenge for the III-V/Si solar cells is the control and suppression of the crystalline defects that arise mainly at the III-V/Si interface due to mismatch of local chemistry. This leads to anti-phase domain (APD) characteristic of the growth of a polar material on a non-polar one, and the microtwins (MT). Indeed, a very high level of structural quality is required so that the efficiency could reach at least $80 \%$ of the theoretical value.

This paper describes our approach in order to obtain a lattice-matched tandem cell composed of a $1.7 \mathrm{eV}$ III-V top and a $1.1 \mathrm{eV}$ c-Si bottom cell and the key material issues that until recently have prevented progress in this field. First promising results are reported on the strategy to analyze and control the structural defects for III-V junction grown on $\mathrm{GaP}$ substrate, displaying a lattice constant close to the silicon. Finally, first very preliminary results obtained on a p-i-n structure grown onto a $\mathrm{GaP}(001)$ substrate are reported.

\section{Monolithic growth of diluted-nitride III-V-N compounds on silicon substrates}

The aim of our work is to provide low-cost and highefficiency tandem cells grown on c-Si substrates, with merging both the monocrystalline $\mathrm{Si}$ approach and the high-efficiency monocrystalline multijunction approach based on III-V materials. We propose to demonstrate the proof-of-concept for a monolithic integration of high-efficiency multijunction CPV device on a low-cost monocrystalline silicon substrate upon which a III-V lattice-matched material is grown using molecular beam epitaxy (MBE). Among binary III-V materials, GaP presents the closest lattice constant to $\mathrm{Si}(0.37 \%$ at $300 \mathrm{~K})$. However, $\mathrm{GaP}$ is not useful due to its large $(2.2 \mathrm{eV})$ and indirect bandgap while a direct bandgap III-V compound is highly required to obtain a thin-film absorber. Yet, nitrogen incorporation in GaP not only enables for a perfect lattice matching on silicon but, with an appropriate adjustment of the $\mathrm{N}$ content (a few percent), also enables GaPN to develop a direct bandgap character due to a strong reduction of the bandgap energy, versus the $\mathrm{N}$ content, which therefore, can act as efficient light absorber (Shan et al. 2000; Xin et al. 2000). Finally, our approach consists of the growth of GaAsPN quaternary compounds, lattice matched on Si, which benefits from As incorporation to enhance the direct bandgap character (Chamings et al. 2008; Robert et al. 2011), while lowering the bandgap down to $1.7 \mathrm{eV}$.

Lattice-matched layers and slightly tilted substrates are used to overcome two of the main difficulties faced by the growth of III-V materials on silicon substrates: misfit dislocations and anti-phase lattice defects, in order to obtain defect-free III-V materials and to get large minority carrier diffusion lengths for the PV applications (Furukawa et al. 2002; Momose et al. 2001; Volz et al. 2011; Létoublon et al. 2011; Grassman et al. 2009). The tandem GaAsPN/Si double-junction solar cell will be electrically connected with a tunnel junction (TJ), either Sibased or III-V based or hybrid (III-V/Si), depending on both the modeling results and the structural properties of the III-V compound. Therefore, the final structure will include a $\mathrm{Si}$ pn junction (1.1 eV gap) grown on $\mathrm{Si}$ substrate, followed by the TJ and the growth of top GaAsPN p-i-n subcell (1.7-1.8 eV). Moreover, prior to any GaAsPN growth onto the silicon, a GaP thin layer has to be grown by MBE to prevent the formation of structural defects.

The state-of-the-art of this approach is epitomized by the National Renewable Energy Laboratory (NREL) which has demonstrated a MOCVD-grown tandem solar cell on a 
Si substrate. The lattice-matched n-i-p solar cell consisted of a $\mathrm{GaP}_{0.98} \mathrm{~N}_{0.02}: \mathrm{Se} / \mathrm{GaAsPN}: 1.8 \mathrm{eV} / \mathrm{GaP}_{0.98} \mathrm{~N}_{0.02}: \mathrm{Zn}$ top cell and a $1.1 \mathrm{eV}$ diffused Si bottom cell, with a GaP-based TJ (Geisz et al. 2005). The growth was done on Si substrates utilizing a miscut of $2^{\circ}$ from the (001) orientated toward (111). Though the dilute III-V nitride addresses the lattice mismatch, the efficiency achieved was about 5.2\% under AM 1.5G (Geisz et al. 2005) (without any antireflection coating), far from the theoretical maximum (Kurtz et al. 1990). This low efficiency was likely due to intrinsic defects in the GaAsPN or in the GaP/Si interface, which limit the minority carrier diffusion length in the absorber inducing a low quantum efficiency in the top cell.

\section{Experimental}

Synchrotron X-ray diffraction has been performed on the D2AM (BM2) CRG beam line at European Synchrotron Radiation Facility (ESRF). A large hybrid pixels detector [14] has been used to allow a much faster data collection than with traditional point detector. This is a crucial point for the acquisition of diffuse signal from crystal defects. The energy has been set at $16 \mathrm{keV}$ in order to limit the air-scattering contribution. The beam size at the sample was about $0.2 \times 0.3 \mathrm{~mm}^{2}$ in vertical and horizontal directions.

TEM has been performed in plan-view and cross-section geometries ([1-1 0] zone axis). Thin plates have been prepared for these two samples by mechanical polishing and ion thinning. The experiment has been performed on a Philips CM30 in high-resolution and conventional modes, with an acceleration voltage of $300 \mathrm{kV}$.

An ultra-high vacuum (UHV) CVD technique has been chosen, for the Si growth. Both thin and thick layers with well-controlled doping levels can be realized with this growth chamber (Quinci et al. 2013). Moreover, due to the use of hydrogenated molecules $\left(\mathrm{SiH}_{4}, \mathrm{GeH}_{4} \ldots\right)$, the presence of hydrogen-terminated surfaces increases the mobility of species at the growth front, which permits low-temperature operation $\left(500-850^{\circ} \mathrm{C}\right.$, i.e. compatible with $\mathrm{GaP}$ epilayers for $\mathrm{Si} / \mathrm{III}-\mathrm{V}$ overgrowth). Lastly, the organization of atomic steps is easier to control as compared to the $\mathrm{H}_{2}$-free $\mathrm{MBE}$ growth. Monodomain (bi-stepped) silicon surface is crucial for subsequent $\mathrm{GaP}$ overgrowth avoiding the formation of APDs.

Concerning the group III-V compounds, we use a MBE chamber which allows to grow samples at low temperature suitable for the diluted-nitrides growth. MBE provides a precise control of the interface, the possibility to operate in far-from-equilibrium thermodynamic conditions. Finally, residual doping in MBE is very low which is mandatory for the development of diluted-nitridebased high-efficiency solar cells. Both growth chambers are linked by a UHV tunnel so that no surface contamination can occur between both $\mathrm{Si}$ and III-V growth steps.

Modeling is performed using the ATLAS device simulator from Silvaco, including the TJ interconnection between the top and the bottom subcells.

\section{Simulations and cell design}

\section{Optimal composition of the GaAsPN compound}

The optimal composition of the GaAsPN has been thoroughly determined since it is necessary to match the $1.7 \mathrm{eV}$ bandgap requirement (or close to) while maintaining a perfect lattice matching between the III-V compound and the Si. To this end, the bandgaps of GaAsPN have been calculated using $\mathrm{sp} \mathrm{s}^{\star} \mathrm{S}_{\mathrm{N}}$ tight-binding calculations adapted for the description of nitrogen incorporation in a diluted regime (Chamings et al. 2008; Robert et al. 2011), as shown in Figure 1 where the solid black line represents the nitrogen composition, as a function of As content, which fulfills the requirement of GaAsPN lattice matched to the silicon substrate. In the same graph, red dotted line gives the calculated bandgap of the corresponding alloy. For instance, the GaAsPN alloy with an As content equal to

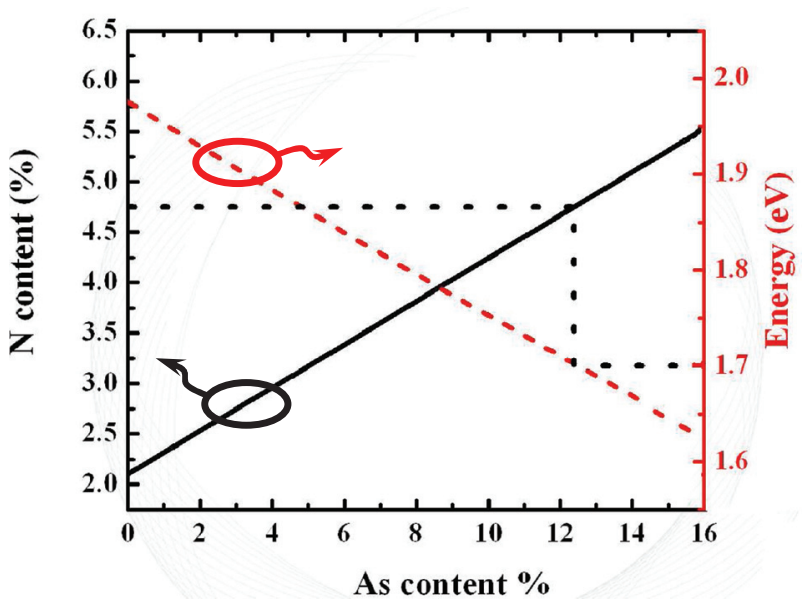

Figure 1 Evolution of the nitrogen content in the GaAsPN alloys as a function of As for an exact lattice matching (black line). Bandgap energy for the corresponding GaAsPN alloys, determined with $\operatorname{sp} 3 \mathrm{~d} 5 \mathrm{~s}^{\star} \mathrm{s}_{\mathrm{N}}$ tight-binding calculations (red line) 
$16 \%$ would need $5.5 \%$ of $\mathrm{N}$ to be lattice matched with $\mathrm{Si}$, giving an alloy with an energy bandgap of $1.63 \mathrm{eV}$. According to this graph, and following the black dotted line, the requirement for a $1.7 \mathrm{eV}$ bandgap is fulfilled with a composition of lattice-matched $\mathrm{GaAs}_{x} \mathrm{P}_{y} \mathrm{~N}_{(1-x-y)}$ equal to $\operatorname{GaAs}_{0.122} \mathrm{P}_{0.83} \mathrm{~N}_{0.048}$.

However, this lattice-matching condition can be slightly overcome without any plastic relaxation of the strain provided that the compound thickness is below a critical thickness. Following the Matthews and Blakeslee formalism (1974) we have determined the critical thickness for the GaAsPN alloy grown onto $\mathrm{Si}$ substrate, assuming a $1-\mu \mathrm{m}$ threshold on critical thickness, and a maximum of $4 \%$ nitrogen (allowing to keep reasonable crystalline quality), the As content is equal to $9 \%$ (Almosni et al. 2013). According to tight-binding calculations, this would give a $1.81 \mathrm{eV}$ bandgap for the $\mathrm{GaAs}_{0.09} \mathrm{P}_{0.87} \mathrm{~N}_{0.04}$ alloy, which is close to the expected $1.7 \mathrm{eV}$ energy bandgap. Therefore, a targeted 1- $\mu \mathrm{m}$-thick absorber 1.7-1.8 eV bandgap GaAsPN layer can be easily grown on silicon substrate, in a pseudomorphic way, according to the critical thickness calculation.

\section{Tunnel junction}

The main approach to connect electrically two cells in a MJSC is to use a two-terminal structure where a TJ, or Esaki diode (Esaki 1958), is placed between the two cells, offering a low resistance due to its high doping $\left(\approx 1.10^{19} \mathrm{~cm}^{-3}\right)$ and good optical transparency due to its low thickness $(\approx 50 \mathrm{~nm})$. To obtain optimal performances, leading to a good voltage addition from the stacked subcells, abrupt dopants profile should be obtained, and thus diffusion of dopants in the TJ should be minimized, and the TJ structure has to be compatible with the overall growth conditions. In particular, using model, two different TJs have been investigated and found suitable for the $1.7 \mathrm{eV} / 1.1 \mathrm{eV}$ targeted tandem cell structure: a hybrid GaP $(n+) / \operatorname{Si}(p+) \mathrm{TJ}$ and a $\operatorname{Si}(n+) / \operatorname{Si}(p+)$ one, considering a $\mathrm{n}$-type $\mathrm{Si}$ absorber in the bottom junction. Considering uniform doping of $5 \times 10^{19} \mathrm{~cm}^{-3}$ on both junction sides (reachable experimentally according to Hall measurements on doped $\mathrm{GaP}$ and Si layers), theoretical current densities as large as $5.5 \times 10^{2} \mathrm{~mA} / \mathrm{cm}^{2}$ in the $\mathrm{GaP}(n+) / \mathrm{Si}$ $(p+)$ TJ case and $4.4 \times 10^{5} \mathrm{~mA} / \mathrm{cm}^{2}$ for the $\mathrm{Si}(n+) / \mathrm{Si}(p+)$ one have been calculated, suitable for the tandem cell application (Rolland et al. 2014). Obviously, the final choice between both will be depending on the actual experimental structural properties attained for each TJ, in particular the maximum doping level, the material structural quality, and the dopant diffusion properties between both III-V and Si materials.

\section{Overall tandem cell structure}

The minority carrier diffusion length in diluted-nitride materials is known to be low, likely due to point defects (Jussila et al. 2014; Dagnelund et al. 2008), spatial localization of carriers due to nitrogen fluctuating composition (Jandieri et al. 2012) or unintentional incorporation of impurities, requiring the use of the collection of fieldaided carriers. Therefore, a thick intrinsic layer is thus used in the current top junction, leading to a PIN top subcell junction. The design of the top GaP/GaAsPN/GaN p-i-n junction (Rolland et al. 2014) has been theoretically studied using Silvaco-ATLAS with carrier mobilities and lifetime values extracted from the literature (Geisz and Friedman 2002; Fahy and O'Reilly 2003), as a function of the GaAsPN absorber thickness. The absorption coefficient value used in the simulation has been extracted from experiments, as shown in the following. As shown in Rolland et al. (2014), an optimal top cell efficiency has been found equal to $9.42 \%$ for a thickness about $1 \mu \mathrm{m}$. This is encouraging since the low carrier mobility and short carrier lifetime have been used in the model, while improvement of the structural quality of the GaP-based diluted-nitride materials is an ongoing research topic to optimize the material quality and reduce the defect density.

\section{Main experimental results and discussion}

\section{Structural defect analyses of the $\mathrm{GaP} / \mathrm{Si}$ interface}

A diluted-nitride compound cannot be grown directly onto the Si substrate, and it is therefore necessary to obtain $\mathrm{GaP} / \mathrm{Si}$ defect-free pseudo-substrates, consisting of a thin GaP layer pseudomorphically grown onto $\mathrm{Si}$, suitable for the subsequent GaAsPN growth. This part describes the key material issues which have prevented until recently the progress in the III-V/Si growth, some analytical development we have used to analyze the structural defects. Finally, some possible routes are proposed to get rid of the defects.

Perfect coherent growth of thin GaP layers onto silicon substrates has already been reported (Nguyen Thanh 
et al. 2012), using both high-resolution transmission electron microscopy (HRTEM) and XRD experiments, without any dislocation misfit. However, two other types of structural defects need to be eliminated, the MTs and the antiphase boundaries (APBs) separating APDs.

XRD analyses are performed on the D2AM BM2 beamline at the ESRF in Grenoble, France. Reciprocal space mappings (RSMs) around the $\mathrm{GaP}(002)$ Bragg reflection have been recorded using a two-dimensional large area hybrid pixel detector (XPAD third generation) (Berar et al. 2009). The RSMs reveal streaks characteristic of the presence of MTs, which are small domains of rotated crystal inside the main GaP phase (Nguyen Thanh et al. 2013). According to the XRD analyses, they are totally suppressed at high-temperature growth (around $580^{\circ} \mathrm{C}$ ) as shown in Figure 2. However, surface roughness is dramatically high at this growth temperature so that growth improvement is necessary to obtain flat surface without any MTs. To this end, we are currently working on different growth recipes, involving a two-step growth strategy (with different growth temperatures) with different growth modes including migration-enhanced epitaxy (MEE), which avoid a 3D GaP-nucleation growth, and MBE. Indeed, a 10-nm-thin GaP layer MEE made at low temperature followed by a 35-nm-thick GaP layer MBE grown at higher temperature already showed very promising results with no evidence of any MTs by XRD experiments and displayed a smaller surface roughness. Details of these results and the growth procedure will be published in a forthcoming paper.

The appearance of APDs is also a key concern of any growth of a polar material, such as GaP, onto a non-polar material, such as silicon. APBs are interfaces between two domains corresponding to each other through the exchange of $\mathrm{Ga}$ and $\mathrm{P}$ atoms. In order to achieve an APD-free GaP layer, the obtaining of a bi-stepped Si surface is required, instead of single-stepped, or self-annihilation of all APDs must be guaranteed (Volz et al. 2011). APD characterization has been performed using X-ray and TEM techniques (Nguyen Thanh et al. 2012).
To obtain a bi-stepped Si surface, we have studied the Si homoepitaxy on substrate having different misorientations along the [110] direction $\left(0^{\circ}, 1^{\circ}, 2^{\circ}, 4^{\circ}\right.$, and $\left.6^{\circ}\right)$, with the same growth conditions. The Si buffer layer surface quality was steadily evaluated through the atomic surface reconstruction using in-situ Reflection HighEnergy Electron Diffraction (RHEED) system and postgrowth Atomic Force Microscopy (AFM) analysis. As a main result, the silicon growth on $6^{\circ}$-off misorientated Si substrates is found to provide the smoother silicon surface, while displaying bi-steps suitable for avoiding any APD formation at the early stages of the growth (Quinci et al. 2013). This is a promising result for the suppression of APD at the GaP/Si interface.

\section{Ohmic contacts on GaP}

Since electrical carriers should be collected efficiently, ohmic contacts constitute an important part of the PV device. Low-resistance ohmic contacts are necessary in order to lower as much as possible any additional effects on the device. Obtaining a good ohmic contact requires the use of an appropriate contact alloy which can be difficult for a p-type large bandgap material such as $\mathrm{GaP}$, for instance. The main properties required for ohmic contacts are linear, symmetrical $I-V$ characteristic and highly reliable and reproducible stable behavior, which imposes an annealing technological procedure. For n-type GaP substrate, we have chosen to use (Ni/ $\mathrm{Au} / \mathrm{Ge}$ ) contact structure, with an optimized annealing temperature at $530^{\circ} \mathrm{C}$, based on the previous results described by Peternai et al. (2003). Ohmic contact with a symmetrical $I-V$ behavior has been obtained with a very good reproducibility of the mechanical stability. Moreover, a low contact resistivity of $10^{-5} \Omega \mathrm{cm}^{2}$ has been obtained on $\mathrm{n}$-GaP substrate $\left(n=1 \times 10^{18} \mathrm{~cm}^{-3}\right)$, which validates the electrical contact. As far as the p-type GaP is concerned, based on the work reported by Baojun Enke, and Fujia (1997) and Zhang et al. (1997)

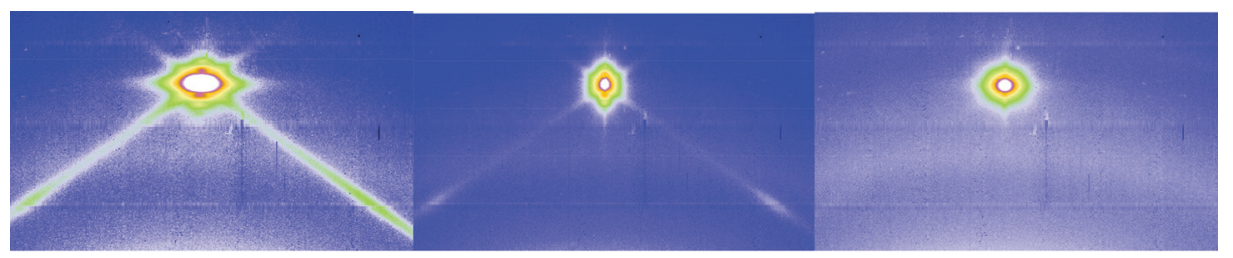

Figure 2 XRD RCM (performed at the ESRF synchrotron) around the (002) reflection on 20-nm-thick GaP/Si samples grown at (left) $350^{\circ} \mathrm{C}$, (middle) $450^{\circ} \mathrm{C}$, and (right) $580^{\circ} \mathrm{C}$. The two streaks underneath the $\mathrm{GaP}(002)$ reflection are characteristics of the MT. The RSMs show the reduction of the MT-induced streaks with the growth temperature increase 
another multimetal film made of $\mathrm{Pd} / \mathrm{Zn} / \mathrm{Pd}$ stacking has been employed, with a higher annealing temperature at $550^{\circ} \mathrm{C}$. As in the n-type doping case, suitable ohmic contact characteristics have been obtained with, again, a very low-specific contact resistivity of $10^{-5} \Omega \mathrm{cm}^{2}$ on pGaP substrate $\left(p=1 \times 10^{18} \mathrm{~cm}^{-3}\right)$.

\section{Preliminary results on heterostructures grown on GaP(001)}

In order to obtain a reproducible incorporation of nitrogen and to improve the optoelectronic properties of our material, a two-step process has been used. This approach has been developed by Harris et al. (2007) on the InGaAsN(Sb) material. It consists in growing the GaAsPN diluted-nitride alloy at low growth temperature $\left(480^{\circ} \mathrm{C}\right)$, which minimizes the point defects generation and any small phase separation (Harris et al. 2007). Then, an annealing step $\left(800^{\circ} \mathrm{C}, 1 \mathrm{~min}\right)$ suppresses some of the remaining point defects usually encountered in the GaPN-based alloys (Jussila et al. 2014), leading to a dramatic improvement of the optical properties (Aho et al. 2013, 2014; Harris et al. 2007). Following this approach, 100-nm-thick GaAsPN layers capped with GaP $(10 \mathrm{~nm})$ have been grown by MBE on $\mathrm{GaP}(001)$ substrate in order to evaluate the diluted-nitride material's structural and optical qualities. The composition has been estimated by cross-modeling results obtained by tightbinding calculation of the GaAsPN energy bandgap as a function of its composition with experimental XRD and PL results that give us access to the lattice parameter and the emission energy, respectively. A $\mathrm{GaAs}_{0.085} \mathrm{P}_{0.90} \mathrm{~N}_{0.015}$ compound lattice matched with the GaP substrate and displaying a $1.78 \mathrm{eV}$ energy photoluminescence (PL) peak position has been obtained. The lattice matching was verified through X-ray diffraction experiments, and PL at room temperature close to the targeted $1.7 \mathrm{eV}$ energy has been obtained (Figure 3). The thickness fringes (also called Pendellösung fringes thickness) that can be seen on the XRD diagram presented in Figure 3 indicate a high crystalline quality along the overall sample thickness. The annealing step has little effect on the XRD diagram figures (not shown here), meaning that the lattice constant remains the same, but has a strong impact on the optical properties as shown in Figure 4, likely due to the nitrogen content homogenization and the health of some point defects not reachable through XRD experiments Aho et al. 2013, 2014; Harris et al. 2007). Indeed, the annealed sample presents a blue-shift of the PL peak energy from 1.78 to $1.9 \mathrm{eV}$, while displaying a 50 times

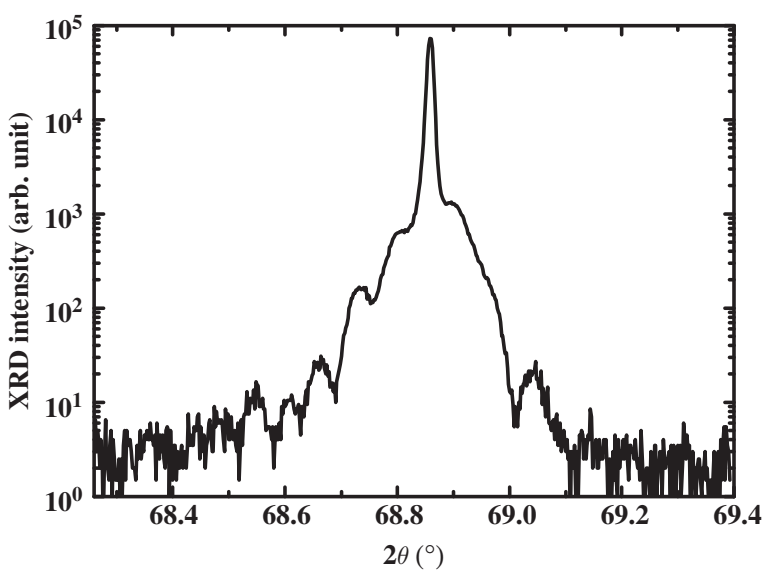

Figure 3 XRD measurement of a 100-nm GaAsPN layer on GaP(001) with a 10-nm GaP cap layer. The XRD pattern has been recorded around the $\mathrm{GaP}(004)$ Bragg reflection

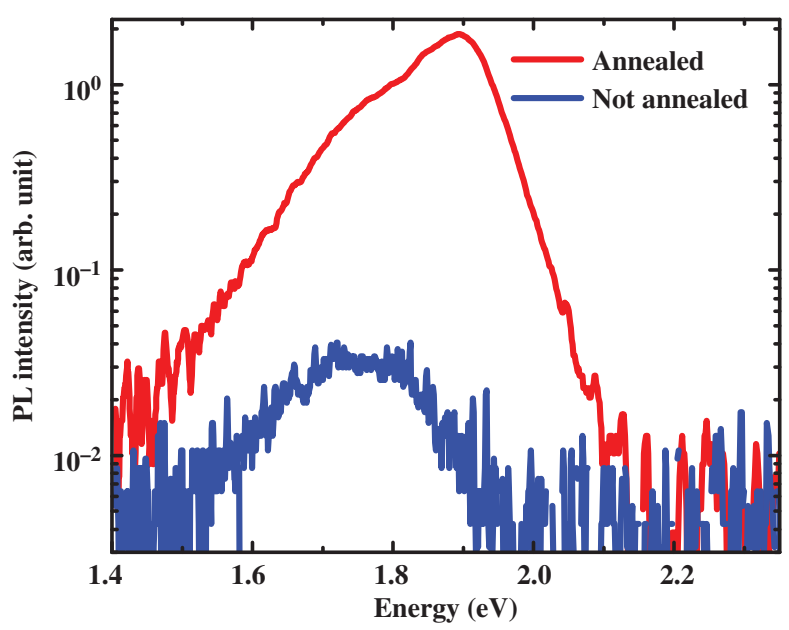

Figure 4 RT PL measurements of a 100-nm GaAsPN layer on GaP (001) with a 10-nm GaP cap layer, before (blue) and after (red) an annealing step $\left(800^{\circ} \mathrm{C}, 1 \mathrm{~min}\right.$.)

enhancement of the PL intensity with a reduction of the FWHM from 264 to $190 \mathrm{meV}$. The low energy tail is the characteristic of the presence of local density of states in the bandgap, typical of $\mathrm{N}$-induced alloy fluctuation and point defects (Zhang et al. 2000). Moreover, the GaAsPN measured absorption spectrum is closer to a direct bandgap material than an indirect bandgap one (Figure 5). The optical properties enhancement upon annealing is well known in the InGaAsN(Sb) system grown at low temperature which reinforces the validity of the two-step process approach for the GaAsPN growth. However, after these preliminary, while promising, results, thorough growth and annealing studies are necessary in order to fully optimize the optical properties of the GaAsPN compound. 


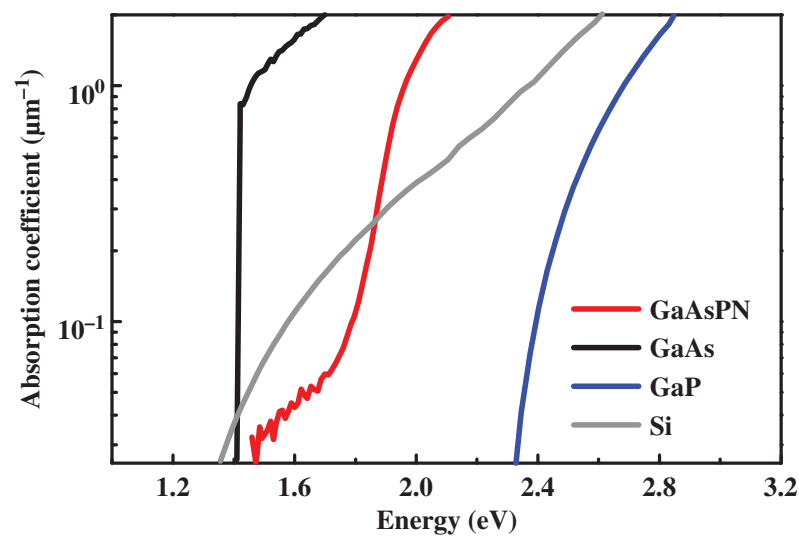

Figure 5 Absorption spectra of the 1- $\mu$ m-thick GaAsPN compound as compared with GaAs direct bandgap, and $\mathrm{GaP}$ and $\mathrm{Si}$ indirect bandgap compounds

These optimizations, along with the use of anti-reflection coating material, constitute a clear pathway for further improvement.

\section{Early-stage results on PIN diodes grown on GaP(001) substrate}

In order to develop the top junction, GaAsPN/GaP PIN diodes have been grown on a n-type $\mathrm{GaP}(001)$ substrate.
The first structure consists of a 300-nm n-doped ( $\mathrm{Si}$ doped, $1 \times 10^{18} \mathrm{~cm}^{-3}$ ) GaP bottom layer, subsequently of a $1-\mu \mathrm{m}$-thick GaAsPN absorber followed by a $250-\mathrm{nm}$ pdoped (Be doped, $1 \times 10^{18} \mathrm{~cm}^{-3}$ ) GaP window layer. The ohmic contacts on the front and back surfaces have been described in section "Ohmic contacts on GaP". The front contact consists of a $5 \times 5 \mathrm{~mm}^{2}$ grid with $10-\mu \mathrm{m}$ large fingers each spaced by $300 \mu \mathrm{m}$. The structure has been annealed at $800^{\circ} \mathrm{C}$ for $1 \mathrm{~min}$ in order to improve the optical properties of the diluted-nitride compounds.

Figure $6(\mathrm{~b})$ shows the $I-V$ characteristic of the diode under an AM1.5G illumination, without any concentration. The efficiency of this diode is $1.6 \%$, which is an encouraging result taking into account that this structure (thickness, growth and composition of the absorbing materials, layer doping, and contacts) has not been fully optimized, so far. Indeed this cell displays a $1.18 \mathrm{~V}$ open-circuit voltage (with theoretical optimal value being around $1.5 \mathrm{~V}$ ) which consists of the state-of-the-art GaAsPN solar cells (see Table 1), with however a low short-circuit current $\left(2.9 \mathrm{~mA} / \mathrm{cm}^{2}\right)$. Figure 6(a) shows the external quantum efficiency (EQE). The absorption edge of the materials corresponds well to the bandgap estimated by PL around $1.9 \mathrm{eV}$ showing that carriers have been extracted from the GaAsPN absorber layer. The absorption edge above $2.2 \mathrm{eV}$ corresponds to the $\mathrm{GaP}$ indirect bandgap. The overall low EQE might be due to

Table 1 Device results review

\begin{tabular}{|c|c|c|c|c|c|c|c|}
\hline Reference & Substrate & PV structure & Absorber thickness $(\mu \mathrm{m})$ & $J_{\mathrm{sc}}\left(\mathrm{mA} / \mathrm{cm}^{2}\right)$ & $V_{\text {oc }}(V)$ & $\mathrm{FF}(\%)$ & $\eta(\%)$ \\
\hline Geisz, Friedman and Kurtz (2002) & GaP & Single PIN & 0.64 & 5.8 & 1.12 & 60 & 3.8 \\
\hline Geisz et al. (2005) & $\mathrm{Si}$ & Tandem & 1 & 6.3 & 1.53 & 54 & 5.2 \\
\hline This work & $\mathrm{GaP}$ & Single PIN & 1 & 2.9 & 1.18 & 51 & 1.6 \\
\hline This work & $\mathrm{GaP}$ & Single PIN & 0.3 & 3.77 & 0.89 & 71 & 2.25 \\
\hline
\end{tabular}
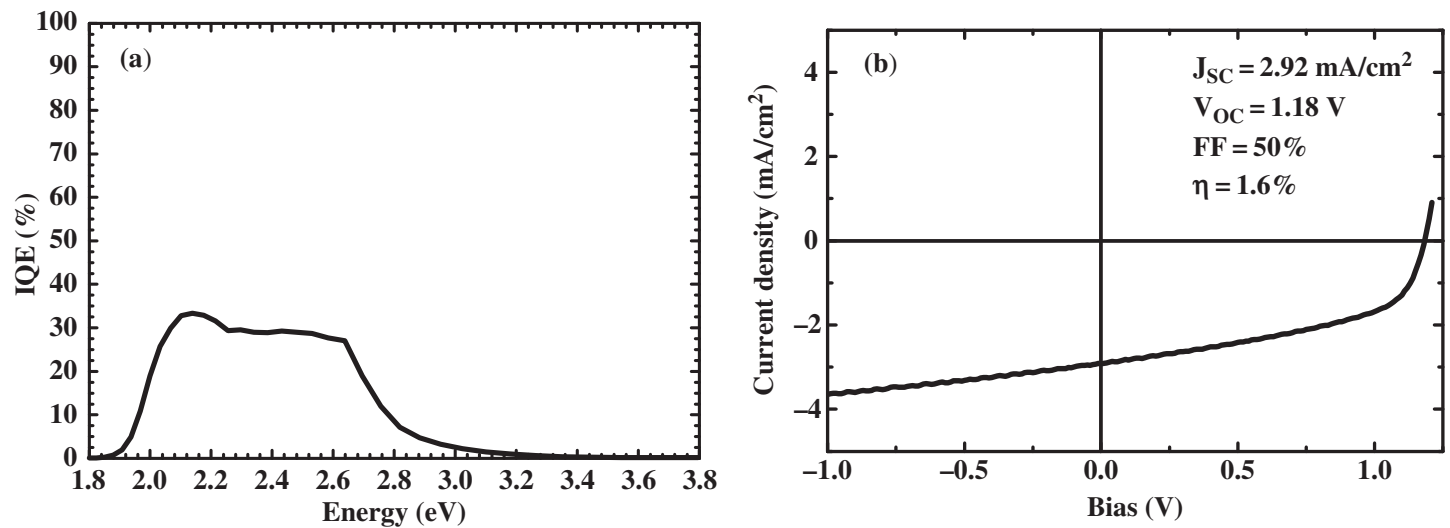

Figure 6 (a) IQE and (b) $J-V$ under unconcentrated AM1.5G solar spectrum of a GaP/GaAsPN $1 \mu \mathrm{m} / \mathrm{GaP}$ PIN junction grown on GaP(001) substrate 
$\mathrm{N}$ localized states and non-radiative traps which reduce the minority carrier diffusion length of the absorber as it has already been reported in diluted-nitride compounds (Geisz, Friedman, and Kurtz 2002) and which could explain the current density increase in the reverse bias regime of the $I-V$ curve (Figure $6(\mathrm{~b})$ ). This low overall EQE might also be due to the presence of $\mathrm{ZnO}$ complex that is almost unavoidable in GaP substrate (Montgomery et al. 2011), therefore reducing the carrier diffusion length. Another issue might be due to the p-GaP layer surface recombination velocity, which could explain the low EQE in the blue region (over 2.6 eV) (Lu et al. 2012).

Therefore, in order to improve the efficiency of those solar cells, we have studied a second structure with a 30$\mathrm{nm}$-thin GaP window layer (as compared to the 250-nmthick previous one), a $0.3-\mu \mathrm{m}$-thick GaAsPN absorber (as compared to the $1-\mu \mathrm{m}$-thick previous one) and a GaP substrate which has been thinned down to $250 \mu \mathrm{m}$ (as compared to the $400-\mu \mathrm{m}$-thick previous one). With this structure, an efficiency of $2.25 \%$, a FF of $71 \%$ (Figure 7(b)), which consists of the state-of-the-art, and an IQE around 35\% (Figure 7(a), under AM1.5G, have been obtained (theoretical maximum of around 16\%), confirming this way for efficiency improvement, even if a systematic study of each individual parameter effect (GaP window layer, low diffusion length of the minority carriers in the absorber, low diffusion length of the carrier in the substrate) still to be done in order to fully interpret the IQE. However, the general issue in this structure is the still low short-circuit current $(3.77 \mathrm{~mA} /$ $\mathrm{cm}^{2}$ ). Recent IQE measurements performed on solar cells with p- and n-doped absorber show an increase of the photon collection with energy above $3 \mathrm{eV}$ for the $\mathrm{n}$-doped sample while the p-doped sample behaves like the PIN structure. This collection increase can be explained by a modification of the space charge region (SCR) position in the solar cells when the absorber is $\mathrm{n}$ doped. This result seems to indicate that the absorber is intrinsically $p$ doped, with a doping density high enough to play a role in the position of the SCR, meaning that the GaAsPN structural properties need an optimization to reduce its intrinsic carrier density. This study will be published in a forthcoming paper. We are currently working on the absorber growth optimization, the annealing step, the GaP passivation layers, and the anti-reflecting coating. Moreover, using front electrical contacts for both $\mathrm{p}$ and $\mathrm{n}$ sides would be necessary to estimate the actual efficiency of this top-junction solar cell, without the GaP substrate influence.

\section{Conclusions}

We have reported promising building blocks for the development of GaAsPN/Si-based dual-junction solar cells. Considering the $\mathrm{GaP} / \mathrm{Si}(001)$ platform development, a good control of the Si initial surface (bi-stepped, smooth, and contaminant-free) for subsequent $\mathrm{GaP}$ overgrowth through Si homoepitaxy on a $6^{\circ}$-off $\mathrm{Si}$ substrate obtained in a UHVCVD-MBE growth cluster has been demonstrated. Such a bi-stepped surface is known to dramatically lower the APD density in the subsequent GaP thin layer growth. We have also demonstrated a MT-free growth of GaP thin layer on $\mathrm{Si}$ at high growth temperature, however with a rough GaP surface. Moreover, a two-step growth strategy showed very promising results with no evidence of any MTs by XRD experiments while displaying a small surface roughness. One of the main issues for the dual-junction solar cell development is obtaining an efficient TJ, modeling of which has shown high theoretical current densities
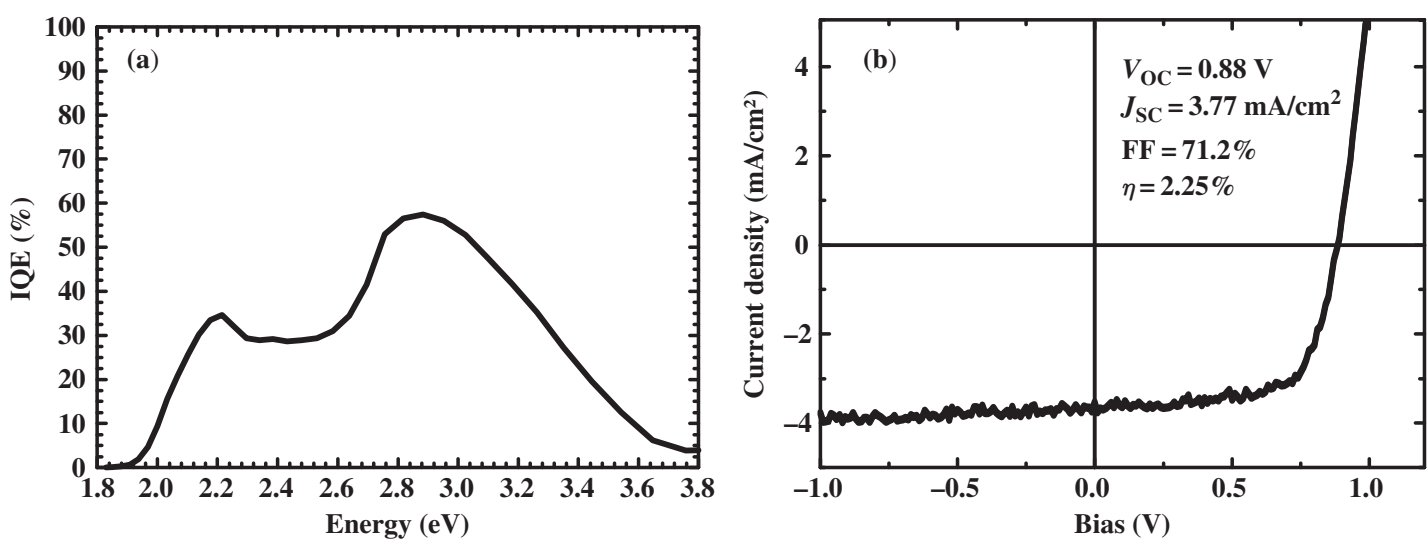

Figure 7 (a) IQE and (b) J-V under unconcentrated AM1.5G solar spectrum, of a GaP/GaAsPN $300 \mathrm{~nm} / \mathrm{GaP}$ PIN junction grown on GaP(001) substrate 
for both $\mathrm{GaP}(n+) / \mathrm{Si}(p+L$ and $\operatorname{Si}(n+) / \operatorname{Si}(p+)$ TJ with doping levels experimentally attained in the GaP alloy, and considering a n-doped $\mathrm{Si}$ bottom absorber. Considering the top-PIN-junction GaAsPN absorber, tightbinding calculation crossed with critical thickness modeling pointed out that a GaAsPN alloy with a composition $9 \%$ of As and $4 \%$ of $\mathrm{N}$ is interesting due to his expected bandgap energy $(1.81 \mathrm{eV})$ and its critical thickness will allow the pseudomorphic growth of a $1-\mu \mathrm{m}$-thick absorber. Therefore, a lattice-matched (with a $\mathrm{GaP}(001)$ substrate) 100-nm-thick GaAsPN alloy has been grown. After a postgrowth annealing step, this alloy displays a strong absorption around 1.8-1.9 eV and efficient PL at room temperature suitable for the targeted solar cell top junction development.

Finally, early-stage GaP/GaAsPN/GaP PIN solar cell prototypes have been elaborated by $\mathrm{MBE}$ on a $\mathrm{GaP}(001)$ substrate. The quantum efficiency (IQE around 30\%) and the $I-V$ curve show that carriers have been extracted from a $1-\mu \mathrm{m}$-thick GaAsPN alloy absorber, with remarkably record open-circuit voltage of $1.18 \mathrm{~V}$. Our best cell was obtained using a 300-nm-thick absorber with 2.25\% efficiency and IQE around 35\%, under AM1.5G. The general issue in this structure is the still low short-circuit current (3.77 $\mathrm{mA} / \mathrm{cm}^{2}$ in our case) meaning that the GaAsPN structural properties need an optimization. A clear pathway to higher efficiency of the top GaAsPN cell would require a thorough optimization of both the MBE growth and the post-growth annealing step, accompanied by a PIN junction architecture improvement. These results are promising and validate our approach for the elaboration of a lattice-matched dual-junction solar cell on silicon substrate.

Acknowledgments: This research was supported by "Région Bretagne" through the PONANT project including FEDER funds. This work was also supported by the French national projects MENHIRS (2011-PRGE-007-01) and SINPHONIC (2011 JS03 006-01).

\section{References}

Aho, A., V. Polojarvi, V. -M. Korpijarvi, J. Salmi, A. Tukiainen, P. Laukkanen, and M. Guina. 2014. "Composition Dependent Growth Dynamics in Molecular Beam Epitaxy of GalnNAs Solar Cells." Solar Energy Materials and Solar Cells 124:150.

Aho, A., A. Tukiainen, V. Polojärvi, J. Salmi, and M. Guina. 2013. "High Current Generation in Dilute Nitride Solar Cells Grown by Molecular Beam Epitaxy." Proceedings of SPIE 8620:86201I-1. doi:10.1117/12.2002972.
Almosni, S., C. Robert, T. Nguyen Thanh, C. Cornet, A. Létoublon, T. Quinci, C. Levallois, M. Perrin, J. Kuyyalil, L. Pedesseau, et al. 2013. "Evaluation of InGaPN and GaAsPN Materials LatticeMatched to Si for Multi-Junction Solar Cells." Journal of Applied Physics 113:123509123509.

Baojun, L., L. Enke, and Z. Fujia. 1997. "Pd/Zn/Pd Ohmic Contacts to p-Type GaP.” Solid-State Electronics 41:917.

Berar, J. -F., N. Boudet, P. Breugnon, B. Caillot, B. Chantepie, J. -C. Clemens, P. Delpierre, B. Dinkespiller, S. Godiot, C. Meessen, et al. 2009. "Methods in Physics Research Section A: Accelerators, Spectrometers." Detectors and Associated Equipment 607:233.

Chamings, J., A. R. Adams, S. J. Sweeney, B. Kunert, K. Volz, and W. Stolz. 2008. "Temperature Dependence and Physical Properties of Ga(NAsP)/GaP Semiconductor Lasers." Applied Physics Letters 93:101108.

Dagnelund, D., I. A. Buyanova, X. J. Wang, W. M. Chen, A. Utsumi, Y. Furukawa, A. Wakahara, and H. Yonezu. 2008. "Formation of Grown-in Defects in Molecular Beam Epitaxial Ga(in)NP: Effects of Growth Conditions and Postgrowth Treatments." Journal of Applied Physics 103:063519. doi:10.1063/1.2895379.

Derkacs, D., R. Jones-Albertus, F. Suarez, and O. Fidaner. 2012. "Lattice-Matched Multijunction Solar Cells Employing a $1 \mathrm{eV}$ GalnNAsSb Bottom Cell." Journal of Photonics for Energy 2:021805.

Esaki, L. 1958. "New Phenomenon in Narrow Germanium p-N Junctions." Physical Review 109:603.

Fahy, S., and E. P. O'Reilly. 2003. "Intrinsic Limits on Electron Mobility in Dilute Nitride Semiconductors." Applied Physical Letters 83:3731. doi:10.1063/1.1622444.

Furukawa, Y., H. Yonezu, K. Ojima, K. Samonji, Y. Fujimoto, K. Momose, and K. Aiki. 2002. "Control of N Content of GaPN Grown by Molecular Beam Epitaxy and Growth of GaPN Lattice Matched to $\mathrm{Si}(100)$ Substrate." Japanese Journal of Applied Physics 41:528-32. doi:10.1143/JJAP.41.528.

Geisz, J. F., and D. J. Friedman. 2002. "lii-N-V Semiconductors for Solar Photovoltaic Applications." Semiconductor Science and Technology 17:769-77. doi:10.1088/0268-1242/17/8/305.

Geisz, J. F., D. J. Friedman, and S. Kurtz. 2002. "GaNPAs Solar Cells Lattice-Matched to GaP." Proceedings of the 29th IEEE Photovoltaics Specialists Conference, New Orleans, Louisiana, 864-7. doi:10.1088/0268-1242/17/8/305.

Geisz, J. F., J. M. Olson, D. J. Friedman, K. M. Jones, R. C. Reedy, and M. J. Romero. 2005. "Lattice-Matched GaNPAs-on-Silicon Tandem Solar Cells." Proceedings of the 31st IEEE Photovoltaics Specialists Conference, Lake Buena Vista, Florida, 695-8. doi:10.1109/PVSC.2005.1488226.

Grassman, T. J., M. R. Brenner, S. Rajagopalan, R. Unocic, R. Dehoff, M. Mills, H. Fraser, and S. A. Ringel. 2009. "Control and Elimination of Nucleation-Related Defects in $\mathrm{GaP} / \mathrm{Si}(001)$ Heteroepitaxy." Applied Physical Letters 94:232106. doi:10.1063/1.3154548.

Harris, J. S., R. Kudrawiec, H. B. Yuen, S. R. Bank, H. P. Bae, M. A. Wistey, D. Jackrel, E. R. Pickett, T. Sarmiento, L. L. Goddard, et al. 2007. "Development of GalnNAsSb Alloys: Growth, Band Structure, Optical Properties and Applications." Physica Status Solidi B 244:2707. doi:10.1002/pssb.200675620.

Jandieri, K., M. K. Shakfa, S. Liebich, M. Zimprich, B. Kunert, C. Karcher, A. Chernikov, K. Volz, W. Stolz, M. Koch, et al. 2012. “Energy Scaling of Compositional Disorder in $\mathrm{Ga}(\mathrm{N}, \mathrm{P}, \mathrm{as}) / \mathrm{GaP}$ 
Quantum Well Structures.” Physical Review B 86:125318. doi:10.1103/PhysRevB.86.125318.

Jussila, H., K. M. Yu, J. Kujala, F. Tuomisto, S. Nagarajan, J. Lemettinen, T. Huhtio, T. O. Tuomi, H. Lipsanen, and M. Sopanen. 2014. "Substitutionality of Nitrogen Atoms and Formation of Nitrogen Complexes and Point Defects in GaPN Alloys." Journalof Physics D: Applied Physics 47:075106. doi:10.1088/0022-3727/47/7/075106.

Kurtz, S. R., P. Faine, and J. M. Olson. 1990. "Modeling of TwoJunction, Series-Connected Tandem Solar Cells Using Top-Cell Thickness as an Adjustable Parameter." Journal of Applied Physics 68:1890. doi:10.1063/1.347177.

Létoublon, A., W. Guo, C. Cornet, A. Boulle, M. Véron, A. Bondi, O. Durand, T. Rohel, O. Dehaese, N. Chevalier, et al. 2011. "X-Ray Study of Antiphase Domains and Their Stability in MBE Grown GaP on Si." Journal of Crystal Growth 323:409-12. doi:16/j. jcrysgro.2010.10.137.

Lu, X., S. Huang, M. B. Diaz, N. Kotulak, R. Hao, R. Opila, and A. Barnett. 2012. "Wide Band Gap Gallium Phosphide Solar Cells.” IEEE Journal of Photovoltaics 2:214-20. doi:10.1109/ JPHOTOV.2011.2182180.

Matthews, J. W., and A. E. Blakeslee. 1974. "Defects in Epitaxial Multilayers: I. Misfit Dislocations." Journal of Crystal Growth 27:118. doi:10.1016/S0022-0248(74)80055-2.

Momose, K., H. Yonezu, Y. Fujimoto, Y. Furukawa, Y. Motomura, and K. Aiki. 2001. "Dislocation-Free and Lattice-Matched $\mathrm{Si} / \mathrm{GaP} 1$ - xNx/Si Structure for Photo-Electronic Integrated Systems." Applied Physics Letters 79:4151. doi:10.1063/ 1.1425451.

Montgomery, K. H., C. R. Allen, I. H. Wildeson, J. H. Jeon, and A. K. Ramdas. 2011. "Gettered GaP Substrates for Improved Multijunction Solar Cell Devices." Journal of Electronic Materials 40:1457. doi:10.1007/s11664-011-1605-1.

Nguyen Thanh, T., C. Robert, W. Guo, A. Létoublon, C. Cornet, G. Elias, A. Ponchet, T. Rohel, N. Bertru, A. Balocchi, et al. 2012. "Structural and Optical Analyses of GaP/Si and (GaAsPN/ GaPN)/GaP/Si Nanolayers for Integrated Photonics on Silicon." Journal of Applied Physics 112:053521. doi:10.1063/1.4751024.

Nguyen Thanh, T., C. Robert, A. Létoublon, C. Cornet, T. Quinci, E. Giudicelli, S. Almosni, N. Boudet, A. Ponchet, J. Kuyyalil, et al. 2013. "Synchrotron X-Ray Diffraction Analysis for Quantitative
Defect Evaluation in GaP/Si Nanolayers." Thin Solid Films 541:36. doi:10.1016/j.tsf.2012.11.116.

Peternai, L., J. Jakabovič, and M. Michalka. 2003. Ohmic Contacts to n-and p-Type GaP." 9th International Workshop on Applied Physics of Condensed Matter, Apcom, 2003, 157-60.

Quinci, T., J. Kuyyalil, T. N. Thanh, Y. P. Wang, S. Almosni, A. Létoublon, T. Rohel, K. Tavernier, N. Chevalier, O. Dehaese, et al. 2013. "Defects Limitation in Epitaxial GaP on Bistepped Si Surface Using UHVCVD-MBE Growth Cluster." Journal of Crystal Growth 380:157-62. doi:10.1016/j.jcrysgro.2013.05.022.

Robert, C., A. Bondi, T. Nguyen Thanh, J. Even, C. Cornet, O. Durand, J. P. Burin, J. M. Jancu, W. Guo, A. Létoublon, et al. 2011. "Room Temperature Operation of GaAsP(N)/GaP(N) Quantum Well Based Light-Emitting Diodes: Effect of the Incorporation of Nitrogen." Applied Physics Letters 98:251110. doi:10.1063/ 1.3601857.

Rolland, A., L. Pedesseau, J. Even, S. Almosni, C. Robert, C. Cornet, J. M. Jancu, J. Benhlal, O. Durand, A. Le Corre, et al. 2014. "Design of a Lattice-Matched lii-V-N/Si Photovoltaic Tandem Cell Monolithically Integrated on Silicon Substrate." Optical and Quantum Electronics 46:1-7. doi:10.1007/s11082-0149909-z.

Shan, W., W. Walukiewicz, K. M. Yu, J. Wu, J. W. Ager, E. E. Haller, H. P. Xin, and C. W. Tu. 2000. "Nature of the Fundamental Band Gap in GaNxP1 - xAlloys.” Applied Physics Letters 76:3251. doi:10.1063/1.126597.

Volz, K., A. Beyer, W. Witte, J. Ohlmann, I. Németh, B. Kunert, and W. Stolz. 2011. "GaP-Nucleation on Exact Si $\left(\begin{array}{lll}0 & 0 & 1\end{array}\right)$ Substrates for III/V Device Integration.” Journal of Crystal Growth 315:37. doi:16/j.jcrysgro.2010.10.036.

Xin, H. P., C. W. Tu, Y. Zhang, and A. Mascarenhas. 2000. "Effects of Nitrogen on the Band Structure of GaNxP1 - xAlloys." Applied Physics Letters 76:1267. doi:10.1063/1.126005.

Zhang, Y., B. Fluegel, A. Mascarenhas, H. P. Xin, and C. W. Tu. 2000. "Optical Transitions in the Isoelectronically Doped Semiconductor GaP:N: An Evolution From Isolated Centers, Pairs, and Clusters to an Impurity Band.” Physics Review $B$ 62:4493. doi:10.1103/PhysRevB.62.4493.

Zhang, F., D. Zhang, B. Li, E. Liu, F. Liu, and R. Gan. 1997. "Pb/Zn/Pd Ohmic Contacts to p-GaP." Materials Science and Engineering B 48:198-201. doi:10.1016/S0921-5107(97)02054-0. 